\title{
On exact special solutions for the stochastic regularized long wave-Burgers equation
}

\author{
Zeliha Korpinar ${ }^{1}$, Mustafa Inc ${ }^{2}$, Ali S. Alshomrani ${ }^{3}$ and Dumitru Baleanu ${ }^{4,5,6^{*}}$ (B)
}

\section{"Correspondence:}

dumitru@cankaya.edu.tr

${ }^{4}$ Department of Mathematics, Cankaya University, 06530 Balgat, Ankara, Turkey

${ }^{5}$ Institute of Space Sciences, Magurele-Bucharest, Romania Full list of author information is available at the end of the article

\begin{abstract}
In this paper, we will analyze the Regularized Long Wave-Burgers equation with conformable derivative (cd). Some white noise functional solutions for this equation are obtained by using white noise analysis, Hermite transforms, and the modified sub-equation method. These solutions include exact stochastic trigonometric functions, hyperbolic functions solutions and wave solutions.

This study emphasizes that the modified fractional sub-equation method is sufficient to solve the stochastic nonlinear equations in mathematical physics.
\end{abstract}

MSC: $35 \mathrm{C07} ; 35 \mathrm{Q} 53$

Keywords: The stochastic RLWBE; White noise analysis; Hermite transforms; The modified sub-equation method

\section{Introduction}

Recently, fractional calculus gained considerable interests and significant theoretical developments in many fields and many studies have been achieved in this field [1-14]. Due to the fact that the stochastic models are more realistic than the deterministic models, we concentrate our study in this paper on the Wick-type stochastic time-fractional Regularized Long Wave-Burgers equation (RLWBE) with conformable derivative (cd). A lot of research on stochastic fractional differential equations has been done recently [1518]. Ghany and Hyder [15] obtained analytical solutions to stochastic time-fractional KdV equations of the Wick-type, Ghany and Zakarya [16] obtained exact traveling wave solutions to a stochastic Schamel KdV equation of Wick-type, in [17] is analyzed a stochastic fractional KdV equation with cd, in [18] is used a white noise functional approach for the fractional coupled $\mathrm{KdV}$ equations and are obtained new soliton solutions. In this paper, we will analyze the time-fractional RLWBE.

The RLWBE with the aid of cd is given by $[19,20]$

$$
\begin{aligned}
& D_{\tau}^{\eta} p(\varkappa, \tau)+\delta D_{x}^{\eta} p(\varkappa, \tau)+\varepsilon(\tau) p(\varkappa, \tau) D_{x}^{\eta} p(\varkappa, \tau)+\lambda(\tau) D_{x x}^{2 \eta} p(\varkappa, \tau) \\
& \quad+\psi D_{x x t}^{3 \eta} p(\varkappa, \tau)=0, \\
& t>0, \quad 0<\eta \leq 1, \\
& (x, \tau) \in \mathbb{R} \times \mathbb{R}_{+}, \quad 0<\eta \leq 1,
\end{aligned}
$$

(c) The Author(s) 2020. This article is licensed under a Creative Commons Attribution 4.0 International License, which permits use, sharing, adaptation, distribution and reproduction in any medium or format, as long as you give appropriate credit to the original author(s) and the source, provide a link to the Creative Commons licence, and indicate if changes were made. The images or other third party material in this article are included in the article's Creative Commons licence, unless indicated otherwise in a credit line to the material. If material is not included in the article's Creative Commons licence and your intended use is not permitted by statutory regulation or exceeds the permitted use, you will need to obtain permission directly from the copyright holder. To view a copy of this licence, visit http://creativecommons.org/licenses/by/4.0/. 
where $\varepsilon(\tau), \lambda(\tau)$ and are limited measurable or integrable functions on $\mathbb{R}_{+} . D_{\tau}^{\eta} p(\varkappa, \tau)$ is the cd operator and $\delta$ and $\psi$ are real valued constants. In [19] are obtained some solutions of this equation by using the modified Kudryashov method. Zhao and Xuan [20] investigated the convergence and existence conditions of solutions for the RLWBE. Bona et al. [21] analyzed the integer ordered type of this equation for an evaluation modeled for water waves. Zhou and Liu [22] obtained kink type waves for the RLWBE. Inan et al. [23] acquired the hyperbolic and trigonometric solutions for this equation. Bas and Kilic [24] obtained the complex solutions by using an algebraic method.

The cd operator was exposed in [25]. This derivative operator can reform the failures of the other definitions. This important operator is the easiest, most natural and effectual definition of the fractional derivative for order $\eta \in(0,1)$. We should note that the definition can be generalized to involve any $\eta$. All the same, the order $\eta \in(0,1)$ is the most influential order.

We say that the conformable fractional differentiability of a function $f:[0, \infty) \mapsto \mathbb{R}$ is nothing else than the classical differentiability. Clearly, the conformable $\eta$-derivative of $f$ at some point $x>0$, where $0<\eta<1$ is the pointwise product $x^{1-\eta} f^{\prime}(x)$ [26].

The cd of order $\eta \in(0,1)$ is described by the following statement [25]:

$$
{ }_{t} D^{\eta} f(t)=\lim _{\vartheta \rightarrow 0} \frac{f\left(t+\vartheta t^{1-\eta}\right)-f(t)}{\vartheta}, \quad f:(0, \infty) \rightarrow \mathbb{R} .
$$

The definition represents a natural formation of normal derivatives. Furthermore, the expression of the definition represents that it is the most natural, and the most effectual definition. The definition for $0 \leq \eta<1$ gives the classical expressions on polynomials.

Several characteristics of the cd are given by [25-27]

(a) ${ }_{t} D^{\eta} t^{\alpha}=\alpha t^{\alpha-\eta}, \forall \eta \in \mathbb{R}$,

(b) ${ }_{t} D^{\eta}(f g)=f_{t} D^{\eta} g+g_{t} D^{\eta} f$,

(c) ${ }_{t} D^{\eta}(f \circ g)=t^{1-\eta} g^{\prime}(t) f^{\prime}(g(t))$,

(d) ${ }_{t} D^{\eta}\left(\frac{f}{g}\right)=\frac{g_{t} D^{\eta} f-f_{t} D^{\eta} g}{g^{2}}$.

This derivative is more advantageous than others because it is easy to apply. Recently, there have been several researches on the conformable form of fractional calculations [27-31].

The stochastic model of Eq. (1.1) in the Wick sense with conformable derivatives can be given in the following process:

$$
D_{\tau}^{\eta} P+(\delta+\varepsilon(\tau) \diamond P) \diamond D_{x}^{\eta} P+\lambda(\tau) \diamond D_{x x}^{2 \eta} P+\psi \diamond D_{x x t}^{3 \eta} P=0,
$$

where " $\diamond$ " is the Wick product on the Kondratiev distribution space $(S)_{-1}, \varepsilon(\tau)$ and $\lambda(\tau)$ are $(S)_{-1}$-valued functions [18].

In order to obtain the exact solutions of the random RLWBE with conformable derivative, we only consider it in a white noise environment, that is, we will discuss the Wick-type stochastic RLWBE (1.2).

Our aim in this work is to obtain a new stochastic soliton and periodic wave solutions of the Wick-type stochastic RLWBE with the aid of cd. We use the modified sub-equation method [32, 33], white noise theory, and Hermite transform to produce a new set of exact soliton and periodic wave solutions for the RLWBE with cd. Moreover, we apply the inverse Hermite transform to obtain stochastic soliton and periodic wave solutions of the Wicktype stochastic RLWBE with the aid of cd. Finally, by an application example, we show how the stochastic solutions can be given as Brownian motion functional solutions. 


\section{Exact solutions of Eq. (1.1)}

In this part, we will investigate exact solutions of RLWBE. Using the Hermite transform of Eq. (1.1), we use the deterministic equation

$$
\begin{gathered}
D_{\tau}^{\eta} \widetilde{P}(\varkappa, \tau, z)+(\widetilde{\delta}(\tau, z)+\widetilde{\varepsilon}(\tau, z) \diamond \widetilde{P}(\varkappa, \tau, z)) \diamond D_{x}^{\eta} \widetilde{P}(\varkappa, \tau, z) \\
+\widetilde{\lambda}(\tau, z) \diamond D_{x x}^{2 \eta} \widetilde{P}(\varkappa, \tau, z)+\widetilde{\psi}(\tau, z) \diamond D_{x x t}^{3 \eta} \widetilde{P}(\varkappa, \tau, z)=0,
\end{gathered}
$$

where $z=\left(z_{1}, z_{2}, \ldots\right) \in\left(\mathbb{C}^{N}\right)_{c}$ is a parameter. To obtain traveling wave solutions to Eq. (2.1), we introduce the transformations $\widetilde{\varepsilon}(\tau, z)=\varepsilon(\tau, z), \widetilde{\lambda}(\tau, z)=\lambda(\tau, z), \widetilde{P}(\varkappa, \tau, z)=p(\varkappa, \tau, z)=$ $p(\xi(\varkappa, \tau, z))$ with

$$
\xi(\varkappa, \tau, z)=k\left(\frac{x^{\eta}}{\eta}\right)+\varpi \int_{a}^{t} \frac{\theta(\tau, z)}{\tau^{1-\eta}} d \tau
$$

where $k, \varpi$ are arbitrary constants and $\theta$ is a nonzero function to be determined. Hence, Eq. (2.1) can be converted to the following NODE:

$$
[\varpi \theta+k(\delta+\varepsilon(\tau, z) p)] \frac{d p}{d \xi}+\lambda(\tau, z) k^{2} \frac{d^{2} p}{d \xi^{2}}+\psi k^{2} \varpi \theta \frac{d^{3} p}{d \xi^{3}}=0
$$

- Considering the solution of Eq. (2.2), we can write it as a series expansion solution as follows:

$$
p(\xi)=\sum_{i=0}^{N} \alpha_{i}(\tau, z) G^{i}(\xi)+\sum_{i=1}^{N} \beta_{i}(\tau, z) G^{-i}(\xi)
$$

where $\alpha_{i}(i=0,1, \ldots, n), \beta_{i}(i=1,2, \ldots, n)$ are functions to be determined later and $G(\xi)$ satisfies the Riccati equation as follows:

$$
G^{\prime}(\xi)=\sigma+G^{2}(\xi)
$$

where $\sigma$ is an arbitrary constants.

- $N$ is obtained with the aid of a balance between the highest order derivatives and the nonlinear terms in Eq. (2.2).

A few special solutions of Eq. (2.4) are given by [34]:

(1) When $\sigma<0$,

$$
G_{1}(\xi)=-\sqrt{-\sigma} \tanh _{\eta}(\sqrt{-\sigma} \xi), \quad G_{2}(\xi)=-\sqrt{-\sigma} \operatorname{coth}_{\eta}(\sqrt{-\sigma} \xi) .
$$

(2) When $\sigma>0$,

$$
G_{3}(\xi)=\sqrt{\sigma} \tan _{\eta}(\sqrt{\sigma} \xi), \quad G_{4}(\xi)=\sqrt{\sigma} \cot _{\eta}(\sqrt{\sigma} \xi)
$$

(3) When $\sigma=0, \rho=$ const.,

$$
G_{5}(\xi)=-\frac{\Gamma(1+\eta)}{\xi^{\eta}+\rho} .
$$


Remark. The generalized trigonometric and hyperbolic functions are defined as [2]

$$
\begin{aligned}
& \tan _{\eta}(\xi)=\frac{E_{\eta}\left(i \xi^{\eta}\right)-E_{\eta}\left(-i \xi^{\eta}\right)}{i\left(E_{\eta}\left(i \xi^{\eta}\right)+E_{\eta}\left(-i \xi^{\eta}\right)\right)}, \quad \cot _{\eta}(\xi)=\frac{i\left(E_{\eta}\left(i \xi^{\eta}\right)+E_{\eta}\left(-i \xi^{\eta}\right)\right)}{E_{\eta}\left(i \xi^{\eta}\right)-E_{\eta}\left(-i \xi^{\eta}\right)} \\
& \tanh _{\eta}(\xi)=\frac{E_{\eta}\left(\xi^{\eta}\right)-E_{\eta}\left(-\xi^{\eta}\right)}{E_{\eta}\left(\xi^{\eta}\right)+E_{\eta}\left(-\xi^{\eta}\right)}, \quad \operatorname{coth}_{\eta}(\xi)=\frac{\left.E_{\eta}\left(\xi^{\eta}\right)+E_{\eta}\left(-\xi^{\eta}\right)\right)}{E_{\eta}\left(\xi^{\eta}\right)-E_{\eta}\left(-\xi^{\eta}\right)}
\end{aligned}
$$

where $E_{\eta}(\xi)=\sum_{i=0}^{N} \frac{\xi^{i}}{\Gamma(1+i \eta)}$ is the Mittag-Leffler function.

By balancing $p \frac{d p}{d \xi}$ with $\frac{d^{3} p}{d \xi^{3}}$ in Eq. (2.2), it is found that $N=2$. Then we can choose the solution of Eq. (2.2) to be given by

$$
p(\xi)=\alpha_{0}+\alpha_{1} G(\xi)+\alpha_{2} G^{2}(\xi)+\beta_{1} G^{-1}(\xi)+\beta_{2} G^{-2}(\xi)
$$

where $G(\xi)$ satisfies Eq. (2.4).

Now, replacing (2.7) and (2.4) into (2.2), by equating all coefficients of $G(\xi)$, we can solve the equations. Then we obtain the following groups of solutions.

One of the obtained these groups is given by

$$
\begin{aligned}
& \alpha_{0}=-\frac{\delta}{\varepsilon(\tau, z)}-\frac{12 k^{2} \lambda(\tau, z)^{2}}{25 \varepsilon(\tau, z)^{2} \alpha_{2}}+\frac{1}{12}\left(\frac{1}{k^{2} \psi}+8 \sigma\right) \alpha_{2}, \\
& \alpha_{1}=-\frac{12 k \lambda(\tau, z)}{5 \varepsilon(\tau, z)}, \quad \beta_{1}=\frac{4 k \lambda(\tau, z)\left(-25 \varepsilon(\tau, z)^{2} \sigma-\frac{36 k^{2} \lambda(\tau, z)^{2}}{\alpha_{2}^{2}}\right)}{125 \varepsilon(\tau, z)^{3}}, \\
& \beta_{2}=\frac{\frac{864 k^{4} \lambda(\tau, z)^{4}}{\varepsilon(\tau, z)^{4}}-\frac{300 k^{2} \lambda(\tau, z)^{2} \sigma \alpha_{2}^{2}}{\varepsilon(\tau, z)^{2}}-625 \sigma^{2} \alpha_{2}^{4}}{6875 \alpha_{2}^{3}}, \\
& \varpi=-\frac{\varepsilon(\tau, z) \alpha_{2}}{12 k \psi} .
\end{aligned}
$$

The exact solutions of Eq. (2.1) are given by:

(1) When $\sigma<0$,

$$
\begin{aligned}
p_{1}(\varkappa, \tau, z)= & -\frac{\delta}{\varepsilon(\tau, z)}-\frac{12 k^{2} \lambda(\tau, z)^{2}}{25 \varepsilon(\tau, z)^{2} \alpha_{2}}+\frac{1}{12}\left(\frac{1}{k^{2} \psi}+8 \sigma\right) \alpha_{2} \\
& +\frac{12 k \lambda(\tau, z)}{5 \varepsilon(\tau, z)} \sqrt{-\sigma} \tanh _{\eta}\left(\sqrt { - \sigma } \left(k\left(\frac{x^{\eta}}{\eta}\right)\right.\right. \\
& \left.\left.-\varpi \int_{a}^{t} \frac{\frac{\varepsilon(\tau, z) \alpha_{2}}{12 k \psi}}{\tau^{1-\eta}} d \tau\right)\right)-\frac{4 k \lambda(\tau, z)\left(-25 \varepsilon(\tau, z)^{2} \sigma-\frac{36 k^{2} \lambda(\tau, z)^{2}}{\alpha_{2}^{2}}\right)}{125 \varepsilon(\tau, z)^{3} \sqrt{-\sigma}} \\
& \times \operatorname{coth}_{\eta}\left(\sqrt{-\sigma}\left(k\left(\frac{x^{\eta}}{\eta}\right)-\varpi \int_{a}^{t} \frac{\frac{\varepsilon(\tau, z) \alpha_{2}}{12 k \psi}}{\tau^{1-\eta}} d \tau\right)\right) \\
& \times \frac{864 k^{4} \lambda(\tau, z)^{4}}{\varepsilon(\tau, z)^{4}}-\frac{300 k^{2} \lambda(\tau, z)^{2} \sigma \alpha_{2}^{2}}{\varepsilon(\tau, z)^{2}}-625 \sigma^{2} \alpha_{2}^{4} \\
& -\operatorname{coth}_{\eta}^{2}\left(\sqrt{-\sigma}\left(k\left(\frac{x^{\eta}}{\eta}\right)-\varpi \int_{a}^{t} \frac{\frac{\varepsilon(\tau, z) \alpha_{2}}{12 k \psi}}{\tau^{1-\eta}} d \tau\right)\right), \\
p_{2}(\varkappa, \tau, z)= & -\frac{\delta}{\varepsilon(\tau, z)}-\frac{12 k^{2} \lambda(\tau, z)^{2}}{25 \varepsilon(\tau, z)^{2} \alpha_{2}}+\frac{1}{12}\left(\frac{1}{k^{2} \psi}+8 \sigma\right) \alpha_{2}
\end{aligned}
$$




$$
\begin{aligned}
& +\frac{12 k \lambda(\tau, z)}{5 \varepsilon(\tau, z)} \sqrt{-\sigma} \operatorname{coth}_{\eta}\left(\sqrt{-\sigma}\left(k\left(\frac{x^{\eta}}{\eta}\right)-\varpi \int_{a}^{t} \frac{\frac{\varepsilon(\tau, z) \alpha_{2}}{12 k \psi}}{\tau^{1-\eta}} d \tau\right)\right) \\
& -\frac{4 k \lambda(\tau, z)\left(-25 \varepsilon(\tau, z)^{2} \sigma-\frac{36 k^{2} \lambda(\tau, z)^{2}}{\alpha_{2}^{2}}\right)}{125 \varepsilon(\tau, z)^{3} \sqrt{-\sigma}} \tanh _{\eta}\left(\sqrt { - \sigma } \left(k\left(\frac{x^{\eta}}{\eta}\right)\right.\right. \\
& \left.\left.-\varpi \int_{a}^{t} \frac{\frac{\varepsilon(\tau, z) \alpha_{2}}{12 k \psi}}{\tau^{1-\eta}} d \tau\right)\right)-\frac{\frac{864 k^{4} \lambda(\tau, z)^{4}}{\varepsilon(\tau, z)^{4}}-\frac{300 k^{2} \lambda(\tau, z)^{2} \sigma \alpha_{2}^{2}}{\varepsilon(\tau, z)^{2}}-625 \sigma^{2} \alpha_{2}^{4}}{6875 \alpha_{2}^{3} \sigma} \\
& \times \tanh _{\eta}^{2}\left(\sqrt{-\sigma}\left(k\left(\frac{x^{\eta}}{\eta}\right)-\varpi \int_{a}^{t} \frac{\frac{\varepsilon(\tau, z) \alpha_{2}}{12 k \psi}}{\tau^{1-\eta}} d \tau\right)\right) .
\end{aligned}
$$

(2) When $\sigma>0$,

$$
\begin{aligned}
& p_{3}(\varkappa, \tau, z)=-\frac{\delta}{\varepsilon(\tau, z)}-\frac{12 k^{2} \lambda(\tau, z)^{2}}{25 \varepsilon(\tau, z)^{2} \alpha_{2}}+\frac{1}{12}\left(\frac{1}{k^{2} \psi}+8 \sigma\right) \alpha_{2} \\
& -\frac{12 k \lambda(\tau, z)}{5 \varepsilon(\tau, z)} \sqrt{\sigma} \tan _{\eta}\left(\sqrt{\sigma}\left(k\left(\frac{x^{\eta}}{\eta}\right)-\varpi \int_{a}^{t} \frac{\frac{\varepsilon(\tau, z) \alpha_{2}}{12 k \psi}}{\tau^{1-\eta}} d \tau\right)\right) \\
& +\frac{4 k \lambda(\tau, z)\left(-25 \varepsilon(\tau, z)^{2} \sigma-\frac{36 k^{2} \lambda(\tau, z)^{2}}{\alpha_{2}^{2}}\right)}{125 \varepsilon(\tau, z)^{3} \sqrt{\sigma}} \cot _{\eta}\left(\sqrt { \sigma } \left(k\left(\frac{x^{\eta}}{\eta}\right)\right.\right. \\
& \left.\left.-\varpi \int_{a}^{t} \frac{\frac{\varepsilon(\tau, z) \alpha_{2}}{12 k \psi}}{\tau^{1-\eta}} d \tau\right)\right)+\frac{\frac{864 k^{4} \lambda(\tau, z)^{4}}{\varepsilon(\tau, z)^{4}}-\frac{300 k^{2} \lambda(\tau, z)^{2} \sigma \alpha_{2}^{2}}{\varepsilon(\tau, z)^{2}}-625 \sigma^{2} \alpha_{2}^{4}}{6875 \alpha_{2}^{3} \sigma} \\
& \times \cot _{\eta}^{2}\left(\sqrt{\sigma}\left(k\left(\frac{x^{\eta}}{\eta}\right)-\varpi \int_{a}^{t} \frac{\frac{\varepsilon(\tau, z) \alpha_{2}}{12 k \psi}}{\tau^{1-\eta}} d \tau\right)\right), \\
& p_{4}(\varkappa, \tau, z)=-\frac{\delta}{\varepsilon(\tau, z)}-\frac{12 k^{2} \lambda(\tau, z)^{2}}{25 \varepsilon(\tau, z)^{2} \alpha_{2}}+\frac{1}{12}\left(\frac{1}{k^{2} \psi}+8 \sigma\right) \alpha_{2} \\
& -\frac{12 k \lambda(\tau, z)}{5 \varepsilon(\tau, z)} \sqrt{\sigma} \cot _{\eta}\left(\sqrt{\sigma}\left(k\left(\frac{x^{\eta}}{\eta}\right)-\varpi \int_{a}^{t} \frac{\frac{\varepsilon(\tau, z) \alpha_{2}}{12 k \psi}}{\tau^{1-\eta}} d \tau\right)\right) \\
& +\frac{4 k \lambda(\tau, z)\left(-25 \varepsilon(\tau, z)^{2} \sigma-\frac{36 k^{2} \lambda(\tau, z)^{2}}{\alpha_{2}^{2}}\right)}{125 \varepsilon(\tau, z)^{3} \sqrt{\sigma}} \tan _{\eta}\left(\sqrt { \sigma } \left(k\left(\frac{x^{\eta}}{\eta}\right)\right.\right. \\
& \left.\left.-\varpi \int_{a}^{t} \frac{\frac{\varepsilon(\tau, z) \alpha_{2}}{12 k \psi}}{\tau^{1-\eta}} d \tau\right)\right)+\frac{\frac{864 k^{4} \lambda(\tau, z)^{4}}{\varepsilon(\tau, z)^{4}}-\frac{300 k^{2} \lambda(\tau, z)^{2} \sigma \alpha_{2}^{2}}{\varepsilon(\tau, z)^{2}}-625 \sigma^{2} \alpha_{2}^{4}}{6875 \alpha_{2}^{3} \sigma} \\
& \times \tan _{\eta}^{2}\left(\sqrt{\sigma}\left(k\left(\frac{x^{\eta}}{\eta}\right)-\varpi \int_{a}^{t} \frac{\frac{\varepsilon(\tau, z) \alpha_{2}}{12 k \psi}}{\tau^{1-\eta}} d \tau\right)\right) .
\end{aligned}
$$

(3) When $\sigma=0, \rho=$ const.,

$$
\begin{aligned}
p_{5}(\varkappa, \tau, z)= & -\frac{\delta}{\varepsilon(\tau, z)}-\frac{12 k^{2} \lambda(\tau, z)^{2}}{25 \varepsilon(\tau, z)^{2} \alpha_{2}}+\frac{1}{12}\left(\frac{1}{k^{2} \psi}+8 \sigma\right) \alpha_{2} \\
& +\frac{12 k \lambda(\tau, z)}{5 \varepsilon(\tau, z)} \frac{\Gamma(1+\eta)}{\left(\left(k\left(\frac{x^{\eta}}{\eta}\right)-\varpi \int_{a}^{t} \frac{\frac{\varepsilon(\tau, z) \alpha_{2}}{12 k \psi}}{\tau^{1-\eta}} d \tau\right)^{\eta}+\rho\right)} \\
& -\frac{4 k \lambda(\tau, z)\left(-25 \varepsilon(\tau, z)^{2} \sigma-\frac{36 k^{2} \lambda(\tau, z)^{2}}{\alpha_{2}^{2}}\right)}{125 \varepsilon(\tau, z)^{3} \Gamma(1+\eta)}\left(\left(k\left(\frac{x^{\eta}}{\eta}\right)\right.\right.
\end{aligned}
$$




$$
\begin{aligned}
& \left.\left.-\varpi \int_{a}^{t} \frac{\frac{\varepsilon(\tau, z) \alpha_{2}}{12 k \psi}}{\tau^{1-\eta}} d \tau\right)^{\eta}+\rho\right) \\
& -\frac{\frac{864 k^{4} \lambda(\tau, z)^{4}}{\varepsilon(\tau, z)^{4}}-\frac{300 k^{2} \lambda(\tau, z)^{2} \sigma \alpha_{2}^{2}}{\varepsilon(\tau, z)^{2}}-625 \sigma^{2} \alpha_{2}^{4}}{6875 \alpha_{2}^{3} \Gamma(1+\eta)^{2}} \\
& \times\left(\left(k\left(\frac{x^{\eta}}{\eta}\right)-\varpi \int_{a}^{t} \frac{\frac{\varepsilon(\tau, z) \alpha_{2}}{12 k \psi}}{\tau^{1-\eta}} d \tau\right)^{\eta}+\rho\right)^{2} .
\end{aligned}
$$

\section{White noise functional solutions of Eq. (1.2)}

In this section, we apply the inverse Hermite transform and Theorem 4.1.1 in [35] to investigate white noise functional solutions of Eq. (1.2). The characteristics of generalized exponential, trigonometric and hyperbolic functions show that there exists a bounded open set $\hat{G} \subset \mathbb{R} \times \mathbb{R}_{+}, a<\infty, b>0$, such that the solution $p(\varkappa, \tau, z)$ of Eq. (2.1) and all its conformable derivatives which are involved in Eq. (2.1) are uniformly bounded for $(\varkappa, \tau, z) \in \hat{G} \times K_{a}(b)$, continuous with respect to $(\varkappa, \tau) \in \hat{G}$ for all $z \in \hat{G} \times K_{a}(b)$ and analytic with respect to $z \in K_{a}(b)$, for all $(\varkappa, \tau) \in \hat{G}$. From Theorem 4.1.1 in [35], there exist $P(\varkappa, \tau) \in(S)_{-1}$ such that $p(\varkappa, \tau, z)=\widetilde{P}(\varkappa, \tau)(z)$ for all $(\varkappa, \tau, z) \in \hat{G} \times K_{a}(b)$ and $P(\varkappa, \tau)$ solves Eq. (1.2) in $(S)_{-1}$. Specially we can choose $\alpha_{2}=\frac{\psi \lambda(\tau)}{\varpi \varepsilon(\tau)}$. Then, by using the inverse Hermite transform for Eqs. (2.7)-(2.11), we will analyze the white noise functional solutions of Eq. (1.2) for $\varepsilon(\tau)>0, \lambda(\tau)>0$ as given below.

(I) Exact stochastic hyperbolic solutions:

$$
\begin{aligned}
& P_{1}(\varkappa, \tau)=-\frac{\delta}{\varepsilon(\tau)}-\frac{12 k^{2} \diamond \lambda(\tau)^{\diamond 2}}{25 \varepsilon(\tau)^{\diamond 2} \diamond\left(\frac{\psi \lambda(\tau)}{\varpi \varepsilon(\tau)}\right)}+\frac{1}{12}\left(\frac{1}{k^{2} \psi}+8 \sigma\right) \diamond \frac{\psi \lambda(\tau)}{\varpi \varepsilon(\tau)} \\
& +\frac{12 k \diamond \lambda(\tau)}{5 \varepsilon(\tau)} \sqrt{-\sigma} \tanh _{\eta}\left(\sqrt{-\sigma}\left(k\left(\frac{x^{\eta}}{\eta}\right)-\varpi \int_{a}^{t} \frac{\frac{\varepsilon(\tau) \diamond\left(\frac{\psi \lambda(\tau)}{\omega \varepsilon(\tau)}\right)}{12 k \diamond \psi}}{\tau^{1-\eta}} d \tau\right)\right) \\
& -\frac{4 k \lambda(\tau) \diamond\left(-25 \varepsilon(\tau)^{2} \diamond \sigma-\frac{36 k^{2} \diamond \lambda(\tau)^{\diamond 2}}{\left(\frac{\psi \lambda(\tau)}{\sigma \varepsilon(\tau)}\right){ }^{\diamond 2}}\right)}{125 \varepsilon(\tau) \diamond^{3} \sqrt{-\sigma}} \operatorname{coth}_{\eta}\left(\sqrt { - \sigma } \left(k\left(\frac{x^{\eta}}{\eta}\right)\right.\right. \\
& \left.\left.-\varpi \int_{a}^{t} \frac{\frac{\varepsilon(\tau) \diamond\left(\frac{\psi \lambda(\tau)}{\omega \varepsilon(\tau)}\right)}{12 k \diamond \psi}}{\tau^{1-\eta}} d \tau\right)\right) \\
& -\frac{\frac{864 k^{4} \diamond \lambda(\tau)^{\diamond 4}}{\varepsilon(\tau)^{\diamond 4}}-\frac{300 k^{2} \lambda(\tau)^{\diamond 2} \diamond \sigma\left(\frac{\psi \lambda(\tau)}{\varpi \varepsilon(\tau)}\right)^{\diamond 2}}{\varepsilon(\tau)^{\diamond 2}}-625 \sigma^{2} \diamond\left(\frac{\psi \lambda(\tau)}{\varpi \varepsilon(\tau)}\right)^{\diamond 4}}{6875\left(\frac{\psi \lambda(\tau)}{\varpi \varepsilon(\tau)}\right)^{\diamond 3} \sigma} \\
& \times \operatorname{coth}_{\eta}^{2}\left(\sqrt{-\sigma}\left(k\left(\frac{x^{\eta}}{\eta}\right)-\varpi \int_{a}^{t} \frac{\frac{\varepsilon(\tau) \diamond\left(\frac{\psi \lambda(\tau)}{\varpi \varepsilon(\tau)}\right)}{12 k \diamond \psi}}{\tau^{1-\eta}} d \tau\right)\right),
\end{aligned}
$$

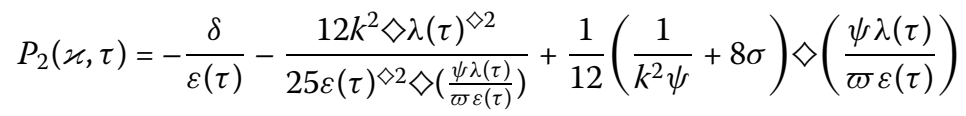

$$
\begin{aligned}
& +\frac{12 k \diamond \lambda(\tau)}{5 \varepsilon(\tau)} \sqrt{-\sigma} \operatorname{coth}_{\eta}\left(\sqrt{-\sigma}\left(k\left(\frac{x^{\eta}}{\eta}\right)-\varpi \int_{a}^{t} \frac{\frac{\varepsilon(\tau) \diamond\left(\frac{\psi \lambda(\tau)}{\sigma \varepsilon(\tau)}\right)}{12 k \diamond \psi}}{\tau^{1-\eta}} d \tau\right)\right) \\
& -\frac{4 k \lambda(\tau) \diamond\left(-25 \varepsilon(\tau)^{2} \diamond \sigma-\frac{36 k^{2} \diamond \lambda(\tau)^{\diamond 2}}{\left(\frac{\psi \lambda(\tau)}{\omega \varepsilon(\tau)}\right)^{\diamond 2}}\right)}{125 \varepsilon(\tau)^{\diamond 3} \sqrt{-\sigma}} \tanh _{\eta}\left(\sqrt { - \sigma } \left(k\left(\frac{x^{\eta}}{\eta}\right)\right.\right.
\end{aligned}
$$




$$
\begin{aligned}
& \left.\left.-\varpi \int_{a}^{t} \frac{\varepsilon(\tau) \diamond\left(\frac{\psi \lambda(\tau)}{\varpi \varepsilon(\tau)}\right)}{12 k \diamond \psi} \frac{\tau^{1-\eta}}{d \tau}\right)\right) \\
& -\frac{\frac{864 k^{4} \diamond \lambda(\tau)^{\diamond 4}}{\varepsilon(\tau)^{\diamond 4}}-\frac{300 k^{2} \lambda(\tau)^{\diamond 2} \diamond \sigma\left(\frac{\psi \lambda(\tau)}{\varpi \varepsilon(\tau)}\right)^{\diamond 2}}{\varepsilon(\tau)^{\diamond 2}}-625 \sigma^{2} \diamond\left(\frac{\psi \lambda(\tau)}{\varpi \varepsilon(\tau)}\right)^{\diamond 4}}{6875\left(\frac{\psi \lambda(\tau)}{\varpi \varepsilon(\tau)}\right)^{\diamond 3} \sigma} \\
& \times \tanh _{\eta}^{2}\left(\sqrt{-\sigma}\left(k\left(\frac{x^{\eta}}{\eta}\right)-\varpi \int_{a}^{t} \frac{\frac{\varepsilon(\tau) \diamond\left(\frac{\psi \lambda(\tau)}{\varpi \varepsilon(\tau)}\right)}{12 k \diamond \psi}}{\tau^{1-\eta}} d \tau\right)\right) .
\end{aligned}
$$

(II) Exact stochastic trigonometric solutions:

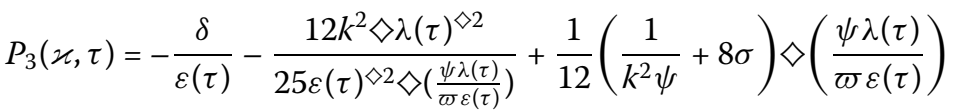

$$
\begin{aligned}
& -\frac{12 k \diamond \lambda(\tau)}{5 \varepsilon(\tau)} \sqrt{\sigma} \tan _{\eta}\left(\sqrt { \sigma } \left(k\left(\frac{x^{\eta}}{\eta}\right)\right.\right. \\
& \left.\left.-\varpi \int_{a}^{t} \frac{\frac{\varepsilon(\tau) \diamond\left(\frac{\psi \lambda(\tau)}{\sigma \varepsilon(\tau)}\right)}{12 k \diamond \psi}}{\tau^{1-\eta}} d \tau\right)\right)+\frac{4 k \lambda(\tau) \diamond\left(-25 \varepsilon(\tau)^{2} \diamond \sigma-\frac{36 k^{2} \diamond \lambda(\tau)^{\diamond 2}}{\left(\frac{\psi \lambda(\tau)}{\omega \varepsilon(\tau)}\right) \diamond 2}\right)}{125 \varepsilon(\tau)^{\diamond 3} \sqrt{\sigma}} \\
& \times \cot _{\eta}\left(\sqrt{\sigma}\left(k\left(\frac{x^{\eta}}{\eta}\right)-\varpi \int_{a}^{t} \frac{\frac{\varepsilon(\tau) \diamond\left(\frac{\psi \lambda(\tau)}{\omega \varepsilon(\tau)}\right)}{12 k \diamond \psi}}{\tau^{1-\eta}} d \tau\right)\right) \\
& +\frac{\frac{864 k^{4} \diamond \lambda(\tau)^{\diamond 4}}{\varepsilon(\tau)^{\diamond 4}}-\frac{300 k^{2} \lambda(\tau)^{\diamond 2} \diamond \sigma\left(\frac{\psi \lambda(\tau)}{\varpi \varepsilon(\tau)}\right) \diamond 2}{\varepsilon(\tau)^{\diamond 2}}-625 \sigma^{2} \diamond\left(\frac{\psi \lambda(\tau)}{\varpi \varepsilon(\tau)}\right)^{\diamond 4}}{6875\left(\frac{\psi \lambda(\tau)}{\varpi \varepsilon(\tau)}\right)^{\diamond 3} \sigma} \\
& \times \cot _{\eta}^{2}\left(\sqrt{\sigma}\left(k\left(\frac{x^{\eta}}{\eta}\right)-\varpi \int_{a}^{t} \frac{\varepsilon(\tau) \diamond\left(\frac{\psi \lambda(\tau)}{\omega \varepsilon(\tau)}\right)}{12 k \diamond \psi} \frac{\tau^{1-\eta}}{1-\eta} \tau\right)\right),
\end{aligned}
$$

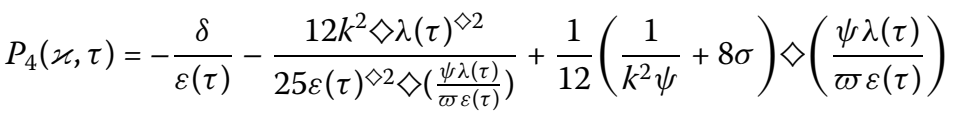

$$
\begin{aligned}
& -\frac{12 k \diamond \lambda(\tau)}{5 \varepsilon(\tau)} \sqrt{\sigma} \cot _{\eta}\left(\sqrt{\sigma}\left(k\left(\frac{x^{\eta}}{\eta}\right)-\varpi \int_{a}^{t} \frac{\frac{\varepsilon(\tau) \diamond\left(\frac{\psi(\tau)}{\omega \varepsilon(\tau)}\right)}{12 k \diamond \psi}}{\tau^{1-\eta}} d \tau\right)\right) \\
& +\frac{4 k \lambda(\tau) \diamond\left(-25 \varepsilon(\tau)^{2} \diamond \sigma-\frac{36 k^{2} \diamond \lambda(\tau)^{\diamond 2}}{\left(\frac{\psi \lambda(\tau)}{\omega \varepsilon(\tau)}\right){ }^{\diamond 2}}\right)}{125 \varepsilon(\tau)^{\diamond 3} \sqrt{\sigma}} \\
& \times \tan _{\eta}\left(\sqrt{\sigma}\left(k\left(\frac{x^{\eta}}{\eta}\right)-\varpi \int_{a}^{t} \frac{\frac{\varepsilon(\tau) \diamond\left(\frac{\psi \lambda(\tau)}{\varpi \varepsilon(\tau)}\right)}{12 k \diamond \psi}}{\tau^{1-\eta}} d \tau\right)\right) \\
& +\frac{\frac{864 k^{4} \diamond \lambda(\tau)^{\diamond 4}}{\varepsilon(\tau)^{\diamond 4}}-\frac{300 k^{2} \lambda(\tau)^{\diamond 2} \diamond \sigma\left(\frac{\psi \lambda(\tau)}{\varpi \varepsilon(\tau)}\right)^{\diamond 2}}{\varepsilon(\tau)^{\diamond 2}}-625 \sigma^{2} \diamond\left(\frac{\psi \lambda(\tau)}{\varpi \varepsilon(\tau)}\right)}{6875\left(\frac{\psi \lambda(\tau)}{\varpi \varepsilon(\tau)}\right)^{\diamond 3} \sigma} \\
& \times \tan _{\eta}^{2}\left(\sqrt{\sigma}\left(k\left(\frac{x^{\eta}}{\eta}\right)-\varpi \int_{a}^{t} \frac{\frac{\varepsilon(\tau) \diamond\left(\frac{\psi \lambda(\tau)}{\varpi \varepsilon(\tau)}\right)}{12 k \diamond \psi}}{\tau^{1-\eta}} d \tau\right)\right) .
\end{aligned}
$$


(III) Exact stochastic wave solutions:

$$
\begin{aligned}
& P_{5}(\varkappa, \tau)=-\frac{\delta}{\varepsilon(\tau)}-\frac{12 k^{2} \diamond \lambda(\tau)^{\diamond 2}}{25 \varepsilon(\tau)^{\diamond 2} \diamond\left(\frac{\psi \lambda(\tau)}{\varpi \varepsilon(\tau)}\right)}+\frac{1}{12}\left(\frac{1}{k^{2} \psi}+8 \sigma\right) \diamond\left(\frac{\psi \lambda(\tau)}{\varpi \varepsilon(\tau)}\right) \\
& +\frac{12 k \diamond \lambda(\tau)}{5 \varepsilon(\tau)} \frac{\Gamma(1+\eta)}{\left(\left(k\left(\frac{x \eta}{\eta}\right)-\varpi \int_{a}^{t} \frac{\varepsilon(\tau) \diamond\left(\frac{\psi \lambda(\tau)}{\frac{1}{2} \varepsilon(\tau)}\right)}{\frac{12 k \Delta \psi}{\tau^{1-\eta}}} d \tau\right)^{\eta}+\rho\right)} \\
& -\frac{4 k \lambda(\tau) \diamond\left(-25 \varepsilon(\tau)^{\diamond 2} \diamond \sigma-\frac{36 k^{2} \diamond \lambda(\tau)^{\diamond 2}}{\left(\frac{\psi \lambda(\tau)}{\omega \varepsilon(\tau)}\right)^{\diamond 2}}\right)}{125 \varepsilon(\tau)^{\diamond 3} \diamond \Gamma(1+\eta)}\left(\left(k\left(\frac{x^{\eta}}{\eta}\right)\right.\right. \\
& \left.\left.-\varpi \int_{a}^{t} \frac{\frac{\varepsilon(\tau) \diamond\left(\frac{\psi \lambda(\tau)}{\omega \varepsilon(\tau)}\right)}{12 k \diamond \psi}}{\tau^{1-\eta}} d \tau\right)^{\eta}+\rho\right) \\
& -\frac{\frac{864 k^{4} \diamond \lambda(\tau)^{\diamond 4}}{\varepsilon(\tau)^{\diamond 4}}-\frac{300 k^{2} \lambda(\tau)^{\diamond 2} \diamond \sigma\left(\frac{\psi \lambda(\tau)}{\omega \varepsilon(\tau)}\right)^{\diamond 2}}{\varepsilon(\tau)^{\curvearrowright 2}}-625 \sigma^{2} \diamond\left(\frac{\psi \lambda(\tau)}{\omega \varepsilon(\tau)}\right)^{\diamond 4}}{6875\left(\frac{\psi \lambda(\tau)}{\varpi \varepsilon(\tau)}\right)^{\diamond 3} \diamond \Gamma(1+\eta)^{2}} \\
& \times\left(\left(k\left(\frac{x^{\eta}}{\eta}\right)-\varpi \int_{a}^{t} \frac{\frac{\varepsilon(\tau) \diamond\left(\frac{\psi(\tau)(\tau)}{\sigma \varepsilon(\tau)}\right)}{12 k \diamond \psi}}{\tau^{1-\eta}} d \tau\right)^{\eta}+\rho\right)^{2} .
\end{aligned}
$$

\section{Example}

In this section, we investigate a special application example to represent the availability of our results and to confirm the real assistance of these results. We explain that the solutions of Eq. (1.2) are strongly dependent on the form of the given functions $\varepsilon(\tau)$ and $\lambda(\tau)$. So, for dissimilar forms of $\varepsilon(\tau)$ and $\lambda(\tau)$, we can find dissimilar solutions of Eq. (1.2) which come from Eqs. (3.1)-(3.5). We illustrate this by giving the following example.

When $\eta=1$,

$$
\begin{aligned}
& \tan _{\eta}(x)=\tan (x), \quad \cot _{\eta}(x)=\cot (x), \quad \tanh _{\eta}(x)=\tanh (x), \\
& \operatorname{coth}_{\eta}(x)=\operatorname{coth}(x), \quad E_{\eta}(x)=\exp (x)
\end{aligned}
$$

Suppose $\lambda(\tau)=\partial \varepsilon(\tau)$ and $\varepsilon(\tau)=f(\tau)+\rho W_{\tau}$, where $\partial$ and $\rho$ are arbitrary constants, $f(\tau)$ is a limited mensurable function on $\mathbb{R}_{+}$and $W_{\tau}$ is the Gaussian white noise which is the time derivative (in the strong sense in $(S)_{-1}$ ) of the Brownian motion $B_{\tau}$. The Hermite transform of $W_{\tau}$ is given by $\widetilde{W}_{\tau}(z)=\sum_{i=0}^{\infty} z_{i} \int_{0}^{t} s \Psi_{i}(\tau) d \tau$ [36]. Using the definition of $\widetilde{W}_{\tau}(z)$, Eqs. (3.1)-(3.5) yield the white noise functional solution of Eq. (1.1) as follows:

$$
\begin{aligned}
P_{1}(\varkappa, \tau)= & -\frac{\delta}{f(\tau)+\rho W_{\tau}}-\frac{12 k^{2} \partial^{2}}{25\left(\frac{\psi \partial}{\varpi}\right)}+\frac{1}{12}\left(\frac{1}{k^{2} \psi}+8 \sigma\right) \frac{\psi \partial}{\varpi} \\
& +\frac{12 k \partial}{5} \sqrt{-\sigma} \tanh \left(\sqrt{-\sigma}\left(k x-\frac{\partial}{12 k}\left\{\int_{a}^{t} f(\tau) d \tau+\rho\left(B_{\tau}-\frac{\tau^{2}}{2}\right)\right\}+c\right)\right) \\
& -\frac{4 k \partial\left(-25 \sigma-\frac{36 k^{2} \varpi^{2}}{\psi^{2}}\right)}{125 \sqrt{-\sigma}} \\
& \times \operatorname{coth}\left(\sqrt{-\sigma}\left(k x-\frac{\partial}{12 k}\left\{\int_{a}^{t} f(\tau) d \tau+\rho\left(B_{\tau}-\frac{\tau^{2}}{2}\right)\right\}+c\right)\right)
\end{aligned}
$$




$$
\begin{aligned}
& -\frac{864 k^{4} \partial \varpi^{3}-300 k^{2} \partial \sigma \psi^{2} \varpi-625 \sigma^{2} \partial \frac{\psi^{4}}{\varpi}}{6875 \psi^{3} \sigma} \\
& \times \operatorname{coth}^{2}\left(\sqrt{-\sigma}\left(k x-\frac{\partial}{12 k}\left\{\int_{a}^{t} f(\tau) d \tau+\rho\left(B_{\tau}-\frac{\tau^{2}}{2}\right)\right\}+c\right)\right), \\
& P_{2}(\varkappa, \tau)=-\frac{\delta}{f(\tau)+\rho W_{\tau}}-\frac{12 k^{2} \partial^{2}}{25\left(\frac{\psi \partial}{\varpi}\right)}+\frac{1}{12}\left(\frac{1}{k^{2} \psi}+8 \sigma\right) \frac{\psi \partial}{\varpi} \\
& +\frac{12 k \partial}{5} \sqrt{-\sigma} \operatorname{coth}\left(\sqrt{-\sigma}\left(k x-\frac{\partial}{12 k}\left\{\int_{a}^{t} f(\tau) d \tau+\rho\left(B_{\tau}-\frac{\tau^{2}}{2}\right)\right\}+c\right)\right) \\
& -\frac{4 k \partial\left(-25 \sigma-\frac{36 k^{2} \varpi^{2}}{\psi^{2}}\right)}{125 \sqrt{-\sigma}} \\
& \times \tanh \left(\sqrt{-\sigma}\left(k x-\frac{\partial}{12 k}\left\{\int_{a}^{t} f(\tau) d \tau+\rho\left(B_{\tau}-\frac{\tau^{2}}{2}\right)\right\}+c\right)\right) \\
& -\frac{864 k^{4} \partial \varpi^{3}-300 k^{2} \partial \sigma \psi^{2} \varpi-625 \sigma^{2} \partial \frac{\psi^{4}}{\varpi}}{6875 \psi^{3} \sigma} \\
& \times \tanh ^{2}\left(\sqrt{-\sigma}\left(k x-\frac{\partial}{12 k}\left\{\int_{a}^{t} f(\tau) d \tau+\rho\left(B_{\tau}-\frac{\tau^{2}}{2}\right)\right\}+c\right)\right), \\
& P_{3}(\varkappa, \tau)=-\frac{\delta}{f(\tau)+\rho W_{\tau}}-\frac{12 k^{2} \partial^{2}}{25\left(\frac{\psi \partial}{\varpi}\right)}+\frac{1}{12}\left(\frac{1}{k^{2} \psi}+8 \sigma\right) \frac{\psi \partial}{\varpi} \\
& -\frac{12 k \partial}{5} \sqrt{\sigma} \tan \left(\sqrt{\sigma}\left(k x-\frac{\partial}{12 k}\left\{\int_{a}^{t} f(\tau) d \tau+\rho\left(B_{\tau}-\frac{\tau^{2}}{2}\right)\right\}+c\right)\right) \\
& +\frac{4 k \partial\left(-25 \sigma-\frac{36 k^{2} \varpi^{2}}{\psi^{2}}\right)}{125 \sqrt{\sigma}} \\
& \times \cot \left(\sqrt{\sigma}\left(k x-\frac{\partial}{12 k}\left\{\int_{a}^{t} f(\tau) d \tau+\rho\left(B_{\tau}-\frac{\tau^{2}}{2}\right)\right\}+c\right)\right) \\
& +\frac{864 k^{4} \partial \varpi^{3}-300 k^{2} \partial \sigma \psi^{2} \varpi-625 \sigma^{2} \partial \frac{\psi^{4}}{\varpi}}{6875 \psi^{3} \sigma} \\
& \times \cot ^{2}\left(\sqrt{-\sigma}\left(k x-\frac{\partial}{12 k}\left\{\int_{a}^{t} f(\tau) d \tau+\rho\left(B_{\tau}-\frac{\tau^{2}}{2}\right)\right\}+c\right)\right), \\
& P_{4}(\varkappa, \tau)=-\frac{\delta}{f(\tau)+\rho W_{\tau}}-\frac{12 k^{2} \partial^{2}}{25\left(\frac{\psi \partial}{\varpi}\right)}+\frac{1}{12}\left(\frac{1}{k^{2} \psi}+8 \sigma\right) \frac{\psi \partial}{\varpi} \\
& -\frac{12 k \partial}{5} \sqrt{\sigma} \cot \left(\sqrt{\sigma}\left(k x-\frac{\partial}{12 k}\left\{\int_{a}^{t} f(\tau) d \tau+\rho\left(B_{\tau}-\frac{\tau^{2}}{2}\right)\right\}+c\right)\right) \\
& +\frac{4 k \partial\left(-25 \sigma-\frac{36 k^{2} \varpi^{2}}{\psi^{2}}\right)}{125 \sqrt{\sigma}} \\
& \times \tan \left(\sqrt{\sigma}\left(k x-\frac{\partial}{12 k}\left\{\int_{a}^{t} f(\tau) d \tau+\rho\left(B_{\tau}-\frac{\tau^{2}}{2}\right)\right\}+c\right)\right) \\
& +\frac{864 k^{4} \partial \varpi^{3}-300 k^{2} \partial \sigma \psi^{2} \varpi-625 \sigma^{2} \partial \frac{\psi^{4}}{\omega}}{6875 \psi^{3} \sigma} \\
& \times \tan ^{2}\left(\sqrt{-\sigma}\left(k x-\frac{\partial}{12 k}\left\{\int_{a}^{t} f(\tau) d \tau+\rho\left(B_{\tau}-\frac{\tau^{2}}{2}\right)\right\}+c\right)\right),
\end{aligned}
$$




$$
\begin{aligned}
P_{5}(\varkappa, \tau)= & -\frac{\delta}{f(\tau)+\rho W_{\tau}}-\frac{12 k^{2} \partial^{2}}{25\left(\frac{\psi \partial}{\varpi}\right)}+\frac{1}{12}\left(\frac{1}{k^{2} \psi}+8 \sigma\right) \frac{\psi \partial}{\varpi} \\
& +\frac{12 k \partial}{5} \frac{1}{\left(\left(k x-\frac{\partial}{12 k}\left\{\int_{a}^{t} f(\tau) d \tau+\rho\left(B_{\tau}-\frac{\tau^{2}}{2}\right)\right\}+c\right)+\rho\right)} \\
& -\frac{4 k \partial\left(-25 \sigma-\frac{36 k^{2} \varpi^{2}}{\psi^{2}}\right)}{125}\left(\left(k x-\frac{\partial}{12 k}\left\{\int_{a}^{t} f(\tau) d \tau+\rho\left(B_{\tau}-\frac{\tau^{2}}{2}\right)\right\}+c\right)+\rho\right) \\
& -\frac{864 k^{4} \partial \varpi^{3}-300 k^{2} \partial \sigma \psi^{2} \varpi-625 \sigma^{2} \partial \frac{\psi^{4}}{\varpi}}{6875 \psi^{3}} \\
& \times\left(\left(k x-\frac{\partial}{12 k}\left\{\int_{a}^{t} f(\tau) d \tau+\rho\left(B_{\tau}-\frac{\tau^{2}}{2}\right)\right\}+c\right)+\rho\right)^{2}
\end{aligned}
$$

where we have already used the following relations [33]:

$$
\begin{aligned}
& \tan ^{\diamond}\left(B_{\tau}\right)=\tan \left(B_{\tau}-\frac{\tau^{2}}{2}\right), \\
& \cot ^{\diamond}\left(B_{\tau}\right)=\cot \left(B_{\tau}-\frac{\tau^{2}}{2}\right), \\
& \tanh ^{\diamond}\left(B_{\tau}\right)=\tanh \left(B_{\tau}-\frac{\tau^{2}}{2}\right), \\
& \operatorname{coth}^{\diamond}\left(B_{\tau}\right)=\operatorname{coth}\left(B_{\tau}-\frac{\tau^{2}}{2}\right) .
\end{aligned}
$$

\section{Final remarks}

We analyzed the RLWBE with cd for deterministic and stochastic forms. In addition, we studied a Wick-model stochastic RLWBE with cd. We investigate some exact solutions with the aid of the modified sub-equation method, Hermite transform and white noise theory. We obtained stochastic hyperbolic and trigonometric wave solutions via the inverse Hermite transform. Furthermore, we investigate an example, to show the stochastic solutions can be obtained as Brownian motion functional solutions. Besides, if $\eta=1$, then the stochastic solutions (4.1)-(4.5) give a new set of stochastic solutions for the Wickmodel stochastic RLWBE with integer derivatives.

This study emphasizes that the modified sub-equation method is sufficient to solve the stochastic nonlinear equations in mathematical physics. The applied method in this paper is a standard, direct, and computerized method, which lets us do confusing and boring algebraic calculations. It is shown that the process can be also applied to other nonlinear stochastic differential equations in mathematical physics.

Acknowledgements

Not applicable.

Funding

Not applicable.

Availability of data and materials

Not applicable. 
Authors' contributions

Each of the authors contributed equally to each part of this work. All authors read and approved the final manuscript.

\section{Author details}

${ }^{1}$ Faculty of Economic and Administrative Sciences, Department of Administration, Mus Alparslan University, Mus, Turkey ${ }^{2}$ Science Faculty, Department of Mathematics, Firat University, 23119 Elazig, Turkey. ${ }^{3}$ Faculty of Science, Department of Mathematics, King Abdulaziz University, 21589 Jeddah, Saudi Arabia. ${ }^{4}$ Department of Mathematics, Cankaya University, 06530 Balgat, Ankara, Turkey. ${ }^{5}$ Institute of Space Sciences, Magurele-Bucharest, Romania. ${ }^{6}$ Department of Medical Research, China Medical University Hospital, China Medical University, Taichung, Taiwan.

\section{Publisher's Note}

Springer Nature remains neutral with regard to jurisdictional claims in published maps and institutional affiliations.

Received: 25 November 2019 Accepted: 28 July 2020 Published online: 20 August 2020

\section{References}

1. Kilbas, A.A., Srivastava, H.M., Trujillo, J.J.: Theory and Applications of Fractional Differential Equations. Elsevier, Amsterdam (2006)

2. Podlubny, I.: Fractional Differential Equation. Academic Press, San Diego (1999)

3. Aslan, E.C., Inc, M.: Optical soliton solutions of the NLSE with quadratic-cubic-Hamiltonian perturbations and modulation instability analysis. Optik 196, 162661 (2019)

4. Samko, S.G., Kilbas, A.A., Marichev, O.I.: Fractional Integrals and Derivatives: Theory and Applications. Gordon \& Breach, New York (1993)

5. Owolabi, K.M., Atangana, A.: High-order solvers for space-fractional differential equations with Riesz derivative. Discrete Contin. Dyn. Syst., Ser. S 12, 567-590 (2019)

6. Tchier, F., Inc, M., Korpinar, Z.S., Baleanu, D.: Solution of the time fractional reaction-diffusion equations with residual power series method. Adv. Mech. Eng. 8(10), 1-10 (2016)

7. Inc, M., Baleanu, D.: Optical solitons for the Kundu-Eckhaus equation with time dependent coefficient. Optik 159 324-332 (2018)

8. Agarwal, P., Al-Mdallal, Q., Je-Cho, Y., Jain, S.: Fractional differential equations for the generalized Mittag-Leffler function. Adv. Differ. Equ. 2018, 58 (2018)

9. Srivastava, H.M., Agarwal, P.: Certain fractional integral operators and the generalized incomplete hypergeometric functions. Appl. Appl. Math. 8(2), 333-345 (2013)

10. Agarwal, P.: Further results on fractional calculus of Saigo operators. Appl. Appl. Math. 7(2), 585-594 (2012)

11. Owolabi, K.M.: Numerical analysis and pattern formation process for space-fractional superdiffusive systems. Discrete Contin. Dyn. Syst., Ser. S 12, 543-566 (2019)

12. Owolabi, K.M., Atangana, A.: Numerical solution of fractional-in-space nonlinear Schrödinger equation with the Riesz fractional derivative. Eur. Phys. J. Plus 131, 335 (2016)

13. Inc, M., Korpinar, Z.S., Al Qurashi, M.M., Baleanu, D.: A new method for approximate solution of some nonlinear equations: residual power series method. Adv. Mech. Eng. 8(4), 1-7 (2016)

14. Korpinar, Z:: On numerical solutions for the Caputo-Fabrizio fractional heat-like equation. Therm. Sci. 22(1), 87-95 (2018)

15. Hossam, A.G., Abd-Allah, H.: Exact solutions for the Wick-type stochastic time-fractional KdV equations. Kuwait J. Sci. 41(1), 75-84 (2014)

16. Hossam, A.G., Abd-Allah, H.: Exact traveling wave solutions for the Wick-type stochastic Schamel KdV equation. Phys. Res. Int. 2014, Article ID 937345 (2014)

17. Hossam, A.G., Abd-Allah, H., Zakarya, M.: Exact solutions of stochastic fractional KdV equation with conformable fractional derivatives. Chin. Phys. B 29(3), 030203 (2020)

18. Hossam, A.G., Okb El Bab, A.S., Zabel, A.M., Abd-Allah, H.: The fractional coupled KdV equations: exact solutions and white noise functional approach. Chin. Phys. B 22(8), 080501 (2013)

19. Korkmaz, A.: Explicit exact solutions to some one dimensional conformable time fractional equations. Waves Random Complex Media 29(1), 124-137 (2019)

20. Zhao, H., Xuan, B.: Existence and convergence of solutions for the generalized BBM-Burgers equations with dissipative term. Nonlinear Anal., Theory Methods Appl. 28, 1835-1849 (1997)

21. Bona, J.L., Pritchard, W.G., Scott, L.R.: An evaluation of a model equation for water waves. Philos. Trans. R. Soc. Lond. A, Math. Phys. Eng. Sci. 302, 457-510 (1981)

22. Zhou, Y., Liu, Q.: Kink waves and their evolution of the RLW-Burgers equation. Abstr. Appl. Anal. 2012, Article ID $109235(2012)$

23. Inan, I.E., Uğurlu, Y., Kılıc, B.: Traveling wave solutions of the RLW-Burgers equation and potential kdv equation by using the-expansion method. J. Sci. Eng. 12(2), 103-110 (2009)

24. Bas, E., Kilic, B.: New complex solutions for rlw Burgers equation, generalized Zakharov-Kuznetsov equation and coupled Korteweg-De Vries equation. World Appl. Sci. J. 11(3), 256-262 (2010)

25. Khalil, R., Al Horani, M., Yousef, A., Sababheh, M.: A new definition of fractional derivative. J. Comput. Appl. Math. 264, 65-70 (2014)

26. Abdelhakim, A.A.: The flaw in the conformable calculus: it is conformable because it is not fractional. Fract. Calc. Appl. Anal. 22(2), 242-254 (2019)

27. Atangana, A., Baleanu, D.: New fractional derivative with nonlocal and non-singular kernel, theory and application to heat transfer model. Therm. Sci. 20(2), 763-769 (2016)

28. Caputo, M., Fabrizio, M.: A new definition of fractional derivative without singular kernel. Prog. Fract. Differ. Appl. 1 73-85 (2015) 
29. Atangana, A., Alkahtani, B.T.: Analysis of non-homogeneous heat model with new trend of derivative with fractional order. Chaos Solitons Fractals 89, 566-571 (2016)

30. He, J.H.: Homotopy perturbation technique. Comput. Methods Appl. Mech. Eng. 178, 257-262 (1999)

31. He, J.H.: Homotopy perturbation method: a new nonlinear analytical technique. Appl. Math. Comput. 135, 73-79 (2003)

32. Ghany, A.H., Okb El Babb, A.S., Zabel, A.M., Hyder, A.: The fractional coupled KdV equations: exact solutions and white noise functional approach. Chin. Phys. B 22, 0805011 (2013)

33. Ghany, A.H., Hyder, A.: Abundant solutions of Wick-type stochastic fractional 2D KdV equations. Chin. Phys. B 23, 0605031 (2014)

34. Zang, S., Zong, Q., Liu, D., Gao, W.: A generalized exp-function method for fractional Riccati differential equations. Commun. Fract. Calc. 1, 48-51 (2010)

35. Holden, H., Øsendal, B., Ubøe, J., Zhang, T.: Stochastic Partial Differential Equations, pp. 159-163. Birkhäuser, Basel (1996)

36. Holden, H., Øsendal, B., Ubøe, J., Zhang, T.: Stochastic Partial Differential Equations. Springer, Berlin (2010)

\section{Submit your manuscript to a SpringerOpen ${ }^{\mathcal{O}}$} journal and benefit from:

- Convenient online submission

Rigorous peer review

Open access: articles freely available online

High visibility within the field

- Retaining the copyright to your article

Submit your next manuscript at $\gg$ springeropen.com 